\title{
Evidenz- und Eminenzbasiertes für ältere Parkinsonpatienten
}

\author{
Erstmals ist eine interdisziplinäre S3-Leitlinie zur Diagnose und Therapie des \\ Parkinson-Syndroms in Vorbereitung. Sie berücksichtigt auch die besonderen \\ Probleme älterer Patienten. Evidenz gibt es dafür aber häufig nicht.
}

\begin{abstract}
Parkinsonpatienten können einiges für sich selbst tun, ist das Credo von Prof. Dr. Walter Maetzler, geriatrisch tätiger Neurologe am Universitätsklinikum Tübingen. Motivation ist gefragt in der Pharmakotherapie aber auch bei der dringend empfohlenen körperlichen und geistigen Aktivität als Alltagsbestandteil ebenso wie in Form einer verordneten Physio-, Ergo- und Logotherapie sowie bei der Ernährung. Die sollte ausgewogen, vitaminund polyphenolreich sein und eine ausreichende Trinkmenge umfassen.
\end{abstract}

\section{Geriatrische Parkinsonpatienten sind anders}

Beim geriatrischen Patienten bestehen neben dem Parkinsonsyndrom noch andere alltagsrelevante Komorbiditäten, die die Lebensqualität mit beeinflussen. Zudem ist bei spätem Erkrankungsbeginn das ganze Gehirn früher und stärker betroffen, die Progression ist schneller, es zeigen sich rascher axiale und nicht-motorische Symptome, seltener Fluktuationen, Dyskinesien, Impulskontrollstörungen und pathologische Tagesmüdigkeit unter dopaminerger Therapie.

\section{Therapie motorischer Symptome}

Evidenz für spezifische Therapieempfehlungen für ältere Pateinten mit fortgeschrittenem Parkinsonsyndrom gibt es wenig, für geriatrische Patienten praktisch keine. Entsprechend enthält die neue S3-Leitlinie nur zwei Empfehlungen für die letztgenannte Patientengruppe.

1. Anticholinergika sind bei geriatrischen Patienten nicht einzusetzen.

2. Bradykinese, Rigor und Tremor sind mit Levodopa und Decarboxylase-

hemmern zu behandeln.

„Wir brauchen das sauberste Levodopa, das wir haben, das führt zu den wenigsten Nebenwirkungen“, sagte Maetzler. Wenn Fluktuationen nicht beherrschbar sind und die Patienten nur schlecht aus den Off-Phasen herauskommen, bei Dyskinesien oder als Add-on-Therapien kommen auch bei Patienten mit fortgeschrittenem Parkinson die anderen Medikamentenklassen zum Einsatz, wobei verstärkt mit Nebenwirkungen zu rechnen ist.

Bei Gabe von Amantadin müssen geriatrische Patienten besonders sorgfältig überwacht werden (Nierenretentionsparameter, Restharn- und EKG-Kontrollen).

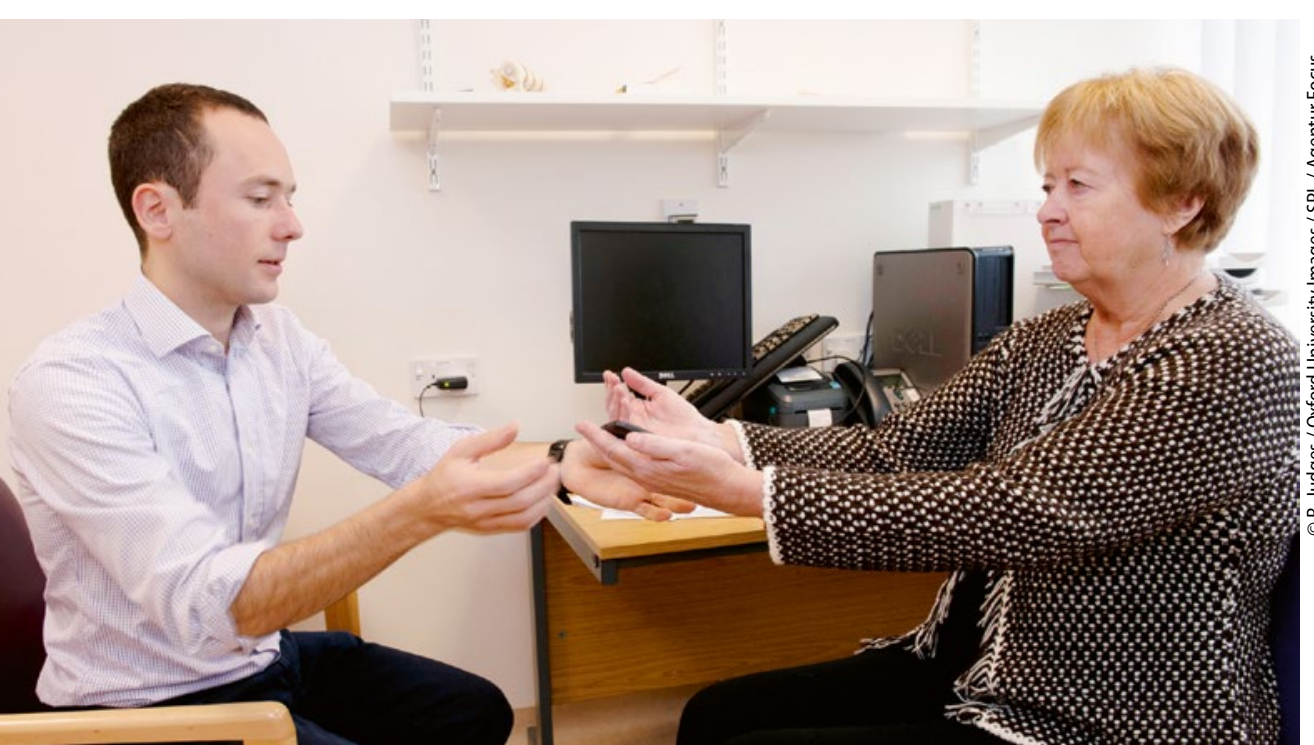

„Außerdem ist Amantadin sehr halluzinogen“, so Maetzler. Dopaminagonisten sind bei kognitiver Leistungseinschränkung, Demenz und/oder psychotischem Erleben nicht einzusetzen.

Gangstörungen sprechen manchmal auf eine erhöhte Dosis L-Dopa an. Bei Gleichgewichtsstörungen kann eine Dosiserhöhung das Symptom jedoch verschlechtern - es ist also Vorsicht geboten.

\section{Therapie nicht-motorischer Symptome}

Eine Demenz bei Parkinsonsyndrom spricht besser auf Cholinesterasehemmer an als eine Alzheimer-Demenz, so Maetzler. Die meisten Daten gebe es für Rivastigmin, einige auch für Donepezil. Die Gabe als Pflaster habe weniger Nebenwirkungen. Bei Halluzinationen bevorzugt er trotz der nötigen Leukozytenkontrolle Clozapin, bei dem bei alten $\mathrm{Pa}$ tienten oft sehr geringe Dosen ausreichen. Quetiapin sei bei diesen Patienten zweite Wahl.

Zur Therapie der Depression haben die Trizyklika zwar den höchsten Empfehlungsgrad (A) erreicht, sind aber aufgrund der anticholinergen Nebenwirkungen für geriatrische Patienten oft ungeeignet. SSRI und Venlafaxin erreichen als Alternativen einen Empfehlungsgrad B.

Schlafstörungen bei fortgeschrittenem Parkinson wurden bisher wenig beachtet. Die Leitlinie empfiehlt bei nächtlicher Akinese und frühmorgendlicher Dystonie transdermales Rotigotin oder retardiertes Ropinirol. Beides kann auch bei Insomnie mit Durchschlafstörungen versucht werden.

- Symposium: Evidenzbasierte Therapie von Syndromen im Alter, 24. 9. 2015, 88. DGN-Kongress, Düsseldorf 23.-26.9.2015 